\title{
DIGNA FAVORE SIMPLICITAS. MITTELALTERLICHE HANDSCHRIFTEN ALS VORLAGE DER NEULATEINISCHEN DICHTUNG DES JOHANNES CAMPANUS UND SEINER SCHÜLER
}

\author{
MARTA VACULÍNOVÁ
}

\section{DIGNA FAVORE SIMPLICITAS. MEDIEVAL MANUSCRIPTS AS A MODEL FOR THE NEO-LATIN POEMS OF HAN CAMPANUS AND HIS STUDENTS}

\begin{abstract}
Latin poets of the early modern period were fond of using prosaic models as themes in their work. The paper examines a unique group of poetic compositions inspired by manuscripts of medieval sermons of professors at the university in Prague. Inspiration was found in them also by Jan Campanus, a professor of history and poetry who encouraged his students to explore historical themes and engage in the study of sources and thus had a significant influence on their work.
\end{abstract}

Keywords: University of Prague - Neo-Latin poetry - Jan Campanus - medieval sermons

DOI: $10.14712 / 23365730.2020 .29$

\section{Handschriften als Quelle der Geschichtsschreibung an der Prager Universität}

Wenn man über das Schicksal alter Handschriften aus den Bibliotheken der Karlsuniversität Prag in der frühen Neuzeit spricht, werden am häufigsten immer wieder die gleichen Stellen aus den edierten Quellen zitiert, die über die Verkäufe der Handschriften berichten oder gar über deren Veräußerung als Makulatur für verschiedene Zwecke. ${ }^{1}$ (Abb. 1) Im Zusammenhang mit den Prager Kollegienbibliotheken spricht man wiederholt von fehlendem Interesse der Propste, die mitunter keinen Unterschied zwischen ihren privaten Bibliotheken und denen der Kollegien machten.

Der Objektivität halber sollten auch andere Stellen zitiert werden, die über die Verbesserung der Situation der Universitätsbibliotheken am Ende des 16. Jahrhunderts berichten, über geplante Katalogisierung, Reinigung der Bibliotheksräume, Sortierung der Bücher und schließlich auch über die nach Erlass des Majestätsbriefes Rudolfs II. erfolgte Universitätsreform, in deren Rahmen die Inventarisierung des Universitätsarchivs und der Bibliotheken

1 Ursprünglich Zikmund WINTER, O životě na vysokých školách pražských knihy dvoje [Zwei Bücher über das Leben an den Prager Hochschulen], Praha 1899, S. 316-317, 376-382. 
durchgeführt wurde. ${ }^{2}$ Aus den Quellen können wir zwar wenig über die Beziehung der Universitätsprofessoren zu den alten Büchern und vor allem Handschriften in den Kollegienbibliotheken erfahren, doch lässt sich anhand der schriftlichen Werke der Professoren und ihrer Studenten einiges feststellen. Es geht vor allem um diejenigen, die sich für Geschichte interessierten.

Den Professoren für Geschichte standen im 16. Jahrhundert zwar einige gedruckte Chroniken zur Verfügung (am häufigsten wurden Johannes Dubravius, Václav Hájek und Martin Kuthen zitiert), viele Quellen zur Geschichte Böhmens waren jedoch nur als Handschriften zugänglich. Wenn wir uns die zweite Ausgabe des Kalenders von Prokop Lupáč anschauen, die ein anderer Geschichtsprofessor, Marek Bydžovský von Florentin, mit einem Verzeichnis der benutzten Quellen versah, stellen wir fest, dass es sich meistens um handschriftliche Überlieferungen handelte, ${ }^{3}$ von denen viele 1602 von Marquard Freher in Hanau herausgegeben wurden. Trotz dieser Ausgabe gab es jedoch andere ungedruckte Quellen, und zwar nicht nur auf Tschechisch, die unsere Autoren in ihren Werken weiterhin zitieren.

Das Geschichtsstudium an der Prager Universität war zu Beginn des 17. Jahrhunderts mit dem Namen von Jan Campanus verbunden. Als Schüler von Marek Bydžovský von Florentin und als dessen Nachfolger im Fach Geschichte zeigte er eifriges Interesse für die Geschichte Böhmens, und seine Universitätsämter machten ihn auch mit der Geschichte der Universität vertraut. Als Propst des Karlskollegs organisierte er während seiner Amtszeit 1605 die Kollegienbibliothek. ${ }^{4}$ Später, als Propst des Allerheiligenkollegs, begann er, die Geschichte dieses Kollegs zusammenzustellen und mit aktuellen Nachrichten zu ergänzen. ${ }^{5}$ Am Anfang dieser Tätigkeit stand wahrscheinlich die Aufgabe, alle Dokumente für den Streit mit dem Allerheiligenkapitel zusammenzutragen. Für seine Arbeit verwendete Campanus Archivmaterial aus dem Karlskolleg und Kodizes aus verschiedenen Kollegienbibliotheken. Diese Erfahrungen nutzte er später bei der Abfassung seines Calendarium beneficiorum, das bis heute eine häufig zitierte Quelle für die Geschichte der Prager Universität und ihrer Bibliotheken ist und als grundlegend für die Prager Universitätsgeschichte gilt. ${ }^{6}$ Aus der Vorrede zum Calendarium erfährt man, dass sich Campanus des Wertes des handgeschriebenen Buches im Mittelalter sehr wohl bewusst war. ${ }^{7}$ Mit Handschriften befassten

2 Ibidem.

3 Prokop LupÁč z Hlavačova, Rerum Boemicarum ephemeris sive Kalendarium historicum, Praha: Jiří Nigrin 1584, S. $\gamma 2 b-\gamma 3 \mathrm{a}$. Schon in der Vorrede zur ersten Ausgabe seiner Ephemeridis von 1578 nennt Lupáč Handschriften vor den gedruckten Büchern als seine Quellen.

4 Acta praepositorum collegii Carolini 1596-1608, Archiv der Karlsuniversität (weiter AUK), 62 B 4, Fol. 437b mit dem Eintrag vom 14. 10. 1605.

5 Mehr darüber Karel BerÁNEK, Mistr Jan Campanus ve správě universitních koleji [Magister Johannes Campanus in der Verwaltung der Universitätskollegs], Praha 1952 (ungedruckte Dissertation), AUK, Sign. 3150.

6 Michal Svatoš, Mistr Jan Kampanus a pražská univerzita [Magister Johannes Campanus und die Prager Universität], Vodňany a Vodňansko 5, 2002, S. 125.

7 Jan Campanus, Calendarium beneficiorum, Praha: Jonata Bohutský 1616, A1b: Sed vilia, inquies, hic multa, praesertim singuli libri, vel singulae sexagenae donatae dicuntur. Videantur sane vilia, dum modo non sint. Nam tales censuum sexagenae quotannis alias pariunt, et libri, praesertim in membrana, ante repertam Typographiam viles non fuere. Das Calendarium wurde herausgegeben und ins Tschechische übersetzt von Karel Hrdina, Mistr Jan Kampanus, Mecenáši Karlovy university [Magister Johannes Campanus. Die Mäzene der Karlsuniversität], Praha 1949, und dann später noch einmal von Josef PoLIŠEnSKÝ - Jana VobrÁTILOVÁ, M. Jana Kampana kalendář dobrodiní, prokázaných pražské Akademii [Des Magisters Johannes Campanus Kalender der Prager Akademie erwiesener Wohltaten], Acta Universitatis Carolinae - Historia Universitatis Carolinae Pragensis (weiter AUC-HUCP) 4, 1963, S. 67-95. 
sich später auch seine Schüler, die Archivquellen bezeugen diese Tätigkeit bei Pavel Ješín und Samuel Martinius. ${ }^{8}$ Als Geschichtsprofessor hielt Campanus Vorlesungen über die Annales Boemorum; ${ }^{9}$ seine annalistische Tätigkeit, mit der er bewusst an die Tradition von Lupáč, Veleslavín und Bydžovský anknüpfte, wurde bisher eher unterschätzt, obwohl ihn dafür seine Zeitgenossen priesen. ${ }^{10}$ Der Grund hierfür liegt darin, dass er seine Annalen in Versform abfasste ${ }^{11}$ und seine Ansichten über Geschichte in verschiedenen Gelegenheitsdichtungen äußerte, als Beispiel kann sein Gedicht an Jiří Dikastus über das fatale Ende der Jahrhunderte dienen. ${ }^{12}$ Obwohl es sich um Gedichte handelt, sollte ihr Informationswert nicht unterschätzt werden.

Bis die umfangreiche Dichtung Campanus' erforscht sein wird, müssen wir uns mit der Ansicht Josef Polišenskýs ${ }^{13}$ begnügen, nämlich dass die Bedeutung von Campanus für die Geschichtsschreibung nicht in seinen eigenen Werken liege, sondern in dem Einfluss auf seine Schüler, die sich später als Historiker und Editoren mittelalterlicher Texte profilierten, wie Pavel Ješín von Bezdězí oder Pavel Stránský. Polišenský nennt drei weitere Studenten, die unter Campanus' Aufsicht ihre Thesen publizierten. ${ }^{14}$ Als Quellen kommen darin meist gedruckte Werke vor, mit Ausnahme von Daniel Machanius, der die Dalimil-Chronik zitiert und darüber hinaus noch eine Handschrift, die er nicht mit Namen nennt, doch stammt sein Zitat aus dem ersten Buch der Königsaaler Chronik, die in der Ausgabe von Freher fehlt und erst Ende des 18. Jahrhunderts publiziert worden ist. ${ }^{15}$

8 Z. WinTER, $O$ životě, S. 382, erwähnt, dass 1617 folgende Handschriften aus Universitätsbesitz an Pavel Ješín ausgeliehen wurden: eine Handschrift in quarto De vita s[anc]ti Adalberti, Vencesilai, Ludomillae, Basilii, Aegyptiacae Mariae; eine andere in quarto, in deren vorderem Teil ein Brief der academia Oxoniensis ad academiam Pragensem enthalten war (das könnte theoretisch die Handschrift NK [= Nationalbibliothek Prag] XI E 3 gewesen sein), ein Pergamentkodex Chronicon de imperatoribus et papis scriptum ad Carolum IV. sowie ein Pergamentfoliant mit der Historia Romana. Ein Jahr später wurde Ješín, der sich Kodizes anschauen wollte, der Zugang zur Bibliothek des Karlskollegs verwehrt. Dafür aber durfte Samuel Martinius 1619 für seinen Vergleich von M. Hieronymus (im Propstbuch des Jan Hus) und Melanchthon in der Bibliothek Material sammeln.

9 M. Svatoš, Mistr Jan Kampanus, S.124.

10 Siehe das Geleitgedicht von Václav RokycanskÝ: Campanum indigito vatem Historicumque Celebrem / Annales patriae, qui tonat ore, suae, in: Jan Žák, Bohemiados rudimenta, Praha: Jiří Hanuš 1610, S. A1b.

11 Er war von Adam Rosacius inspiriert, der 1591 seine Tempestates veröffentlichte (s. Lucie StorchovÁ, Paupertate styloque connecti. Utváreni humanistické učenecké komunity v českých zemích [Paupertate styloque connecti. Die Bildung humanistischer Gelehrtengemeinschaften in den böhmischen Ländern], Praha 2011, S. 195).

12 De centenariorum exitu plerumque turbulento in der Gedichtsammlung Sedecimi a partu virgineo centenarii triennium postremum, Praha 1601 (Antonín TruHLÁř - Karel Hrdina - Jan MARTíneK - Josef HeJnic, Rukovět' humanistického básnictvív Čechách a na Moravě [Vademecum der humanistischen Dichtkunst in Böhmen und Mähren], weiter RHB, hier RHB I, Praha 1962, S. 261).

13 Josef PolišEnskÝ, České dějepisectvi predbělohorského obdobi a pražská akademie [Die tschechische Geschichtsschreibung des 16. und 17. Jahrhunderts und die Prager Akademie vor 1620], AUC-HUCP 4/2, 1963, S. 115-137.

14 J. Polı̌̌EnSKÝ, ibidem, nennt auf Seite 132 die gedruckten Quellen, lässt jedoch die Handschriften aus.

15 Bernard Machanius, Theses ex historia regum Bohemiae, Praha: Matouš Pardubický 1613, S. B3b über die Vermählung von Wenzel III. mit Viola von Teschen: „quae copula in principio laeticiam habuit, in medio maestitiam, in fine conclusionem heu non bonam!“ Josef EMLER (Hg.), Petra Žitavského kronika Zbraslavská [Die Königsaaler Chronik des Peter von Zittau], in: Fontes rerum Bohemicarum, IV, Praha 1882-1884, S. 106. Der Kommentar des Chronisten bezieht sich freilich auf die Vermählung von Wenzels Schwester Anna mit Heinrich von Kärnten ein Jahr später. Mehr dazu Marie BlÁHovÁ, Osudy Zbraslavské kroniky [Die Geschicke der Königsaaler Chronik], Studia historica Brunensia 62, 2015, S. 143-154. 
Wie gesagt, bevorzugte Campanus die Versform und veranlasste seine Schüler, Gedichte mit historischer Thematik zu schreiben. Es handelte sich vor allem um Regionalgeschichte, die für Anfänger eine weniger komplizierte Materie bot und darüber hinaus in den böhmischen Städten eine lange Tradition hatte. Eher abneigend stellte sich Campanus gegen die sogenannten Herrscherreihen. ${ }^{16}$ Als Vorlage für diese Dichtungen wurden meistens gedruckte Quellen benutzt, doch sollten die Studenten auch handgeschriebene Quellen aus Privatbibliotheken nutzen. ${ }^{17}$ In einem Gedicht beklagt sich Campanus darüber, dass seine Studenten über die Geschichte Böhmens zwar schreiben, aber das Geschriebene nicht publizieren wollen. ${ }^{18}$ Wir können also davon ausgehen, dass viel mehr Dichtungen dieser Art, als heute bekannt, unter seiner Aufsicht entstanden sind.

\section{Mittelalterliche Handschriften als Inspiration für neulateinische Gedichte}

Eine andere Art von Poesie, die Thema dieses Beitrags ist, stellen Bearbeitungen von Archivalien und mittelalterlichen Handschriften aus Universitätsbesitz dar. Sie sind sämtlich mit Johann Campanus entweder als Dichter oder als Professor verbunden. Bevor wir grundsätzliche Fragen erörtern, geben wir eine kurze Übersicht über die relevanten Dichtungen:

1. Andreas Rochotius, Проvo $\mu$ í $\alpha$ scholarium ex authent: habita C. ne filius pro patre, Praha: Jiří Hanuš 1612. Das in Verse gesetzte Schutzprivileg für Studenten des Kaisers Friedrich Barbarossa, kürzlich gefunden und zum Vorschein gebracht.

2. Jan Campanus, Elegia de admiranda et nunquam satis decantata Christi nativitate (weiter De nativitate), Praha: Pavel Sessius 1618. Elegie über die Geburt Christi, Neujahrsgeschenk für Freunde, inspiriert durch eine Predigt von Peter Koněprus aus einer Handschrift der Kollegienbibliothek der Böhmischen Nation.

3. Jan Campanus, Querela pacis bellico tumultu Bohemiam anno 1618 inquietante meditata, Praha: Pavel Sessius 1618. Die Klage des Friedens im Jahre 1618 zu Beginn des Böhmischen Krieges, inspiriert durch eine Predigt von Simon von Tišnov. Vorlage: NK XIII F 16, Fol. 111r-113v.

4. Jan Campanus, Elegidion de Domini epiphania (weiter De epiphania), Praha: Pavel Sessius 1618. Neujahrsgeschenk für das Jahr 1618, inspiriert teilweise durch eine gleichnamige Predigt von Stephan von Páleč. Vorlage: NK VIII F 2, Fol. 120v-130v.

5. Jan Campanus, Nemo vir perfectus, Praha: Pavel Sessius 1618. Ausgabe der Vita des heiligen Nemo vom selben Jahr. Campanus entschuldigt sich in den Paratexten mehrmals

16 Vergl. das Einführungsgedicht von Campanus in der gereimten Chronik der Stadt Saaz von Václav RIPA, Lucko, seu principatus urbis Zacae, Praha: Daniel Sedlčanský 1605, S. A7a: „Nec tamen irrumpis neque cunctos ordine reges / atque duces versum cogis inire tuum. / Exiguo primum tua cymbula aequore ludit / conditur imparibus dum tibi Luczko modis.“

17 In seinem Vorwort zu Lucko schreibt Václav Ripa, dass er es nicht wagte, die angesehenen Bürger von Saaz um ihre Handschriften zu bitten, S. A5b: ,plurima fors peculiaria Chirographa quae manu doctissimorum Virorum, posteritatique pospicientium, conscripta Zacae in Bibliothecis illorum diligenter asservantur, me longius inde degentem, defecerint".

18 V. RipA, Lucko, S. A7a: „Scribite dicebam Bojemidos arma Boemi / Nam pius est Patriae facta referre labor. / Scripserunt plures, sed nemo ex ordine tanto, / Qui sua sub lucem mittere vellet, erat. / Unus (et hoc maior tua Gloria) RIPA monentis / Ne frustra fuerint, tot mea dicta, caves.“ 
dafür, dass er den Text nicht in Versen, sondern in Prosa den Lesern nahebringt, und fordert seinen ehemaligen Schüler Martin Mylius auf, ein Gedicht daraus zu machen.

Die zweite Auflage folgte schon 1619 und wurde von dem Universitätspedell Matěj Bohuslav Jičínský herausgegeben.

Schon im Exil brachte Tobias Hauschkonius, ehemaliger Schüler von Campanus und sein Nachfolger auf dem Gebiet der Psalmendichtung, in seinem Buch Pensum sacrum academico-evangelicum, Dresden: heredes Gimelis Bergen 1638, zwei mittelalterliche gereimte Dichtungen aus dem Alten und Neuen Testament heraus, die aus einer Handschrift der Prager Universität abgeschrieben sind. Abdrucke älterer Universitätsprivilegien veröffentlichte ein anderer Schüler von Campanus im Exil, Samuel Martinius, in seiner Schrift Parentatio. ${ }^{19}$

\section{Identifizierung der Handschriften und Vergleich der Vorlagen und der Gedichte}

Um die Art der Verwandlung der Prosavorlage in ein Gedicht feststellen zu können, war es erforderlich, die Handschriften aus dem Besitz der Karlsuniversität zu identifizieren. Dies ist uns bei der Vorlage von Querela pacis gelungen. Die Predigt von Simon von Tišnov zum Thema Rogate, quae ad pacem sunt, hat sich in einem einzigen Exemplar erhalten, und zwar in einer Handschrift aus dem ehemaligen Kolleg der Böhmischen Nation, die sich heute in der Nationalbibliothek in Prag unter der Signatur XIII F 16, Fol. 111r-113v befindet. Identifizierbar ist auch die Vorlage von De epiphania aus der Bibliothek des Karlskollegs, ${ }^{20}$ Signatur der Nationalbibliothek NK VIII F 2, Fol. 120v-130v (Incipit: Apertis thesauris suis obtulerunt ei munera). Verschollen ist die Handschrift mit einer Predigt des Peter von Koněprus, die jedoch in dem mittelalterlichen Bücherverzeichnis des Kollegs der Böhmischen Nation unter der Signatur P 41 auffindbar ist. ${ }^{21}$ Die dichterische Übertragung von Campanus ist dementsprechend die einzige Information über den Predigtinhalt zum Thema Novum faciet Dominus supra terram: Faemina circumdabit virum. ${ }^{22}$ Der Text der Nemo-Vita war im Mittelalter in Böhmen sehr populär ${ }^{23}$ und in der Nationalbibliothek in Prag werden mehrere Abschriften davon aufbewahrt. ${ }^{24}$ Ihr Vergleich mit der Ausgabe von 1618 führte letztlich zu dem Schluss, dass keine von ihnen Campanus als Vorlage dienen

19 Samuel Martinius, Parentatio antiquissimae ... academiae Carolinae, Wittenberg: Christian Tham 1624, S. B7b-B8b Kuttenberger Dekret, S. D8ab Mandat Karls von Liechtenstein für die Übergabe der Karlsuniversität an das Jesuitenkolleg im Klementinum im Jahre 1622.

20 Emma Urbánková, Zbytky knihovny M. Václava Korandy ml. v Universitní knihovně v Praze [Reste der Bibliothek Magister Wenzel Korandas d.J. in der Universitätsbibliothek Prag], in: Ročenka Universitní knihovny v Praze 1956, S. 147.

21 Ausgabe des Katalogeintrags in: Zuzana Silagiová - František ŠmaHel (edd.), Catalogi librorum vetustissimi Universitatis Pragensis / Die ältesten Bücherkataloge der Prager Universität, in: Corpus Christianorum Continuatio Mediaevalis 271: Magistri Iohannis Hus Opera omnia, tomus XXXVIIB - Supplementum II, Turnhout 2015, S. 121-123.

22 Jeremia $31,22$.

23 Vgl. Lucie DoležAlová, Absolute Alterity in the Cult of Saints: Saint Nobody, in: Ada Marinković - Trpimir Vedriš (eds.), Identity and Alterity in Hagiography and the Cult of Saints, Zagreb 2010, S. 89-102.

24 NK XI E 6, Fol. 93rv; XIII G 18, Fol. 262v-263r; III E 27, Fol. 63r-64r; für andere Bibliotheken vgl. L. DoLEŽAlovÁ, Absolute Alterity, S. 95-96. 
konnte. Der Text der Ausgabe kommt der Variante in der Handschrift der Österreichischen Nationalbibliothek Cod. 3282, Fol. 5r-7v am nächsten, ist jedoch nicht völlig identisch. ${ }^{25}$ Die Vorlage für Rochotius war in der Nationalbibliothek nicht auffindbar. Er selbst schreibt in der Vorrede nicht genau, wo er die ,von Schimmel und Motten“" umgebene Handschrift gefunden hatte. ${ }^{26} \mathrm{Da}$ er seine Thesen Ende 1609 schrieb, können wir annehmen, dass es sich bei ihr um eine Abschrift handelte, die während der Inventarisierung der Universitätsprivilegien zum Vorschein kam - und wahrscheinlich aussortiert wurde, da sie keinen direkten Bezug zur Prager Universität hatte. Der Text des Privilegs ist wohl bekannt und war es auch damals, er wurde in das Corpus iuris civilis einverleibt und war an der Schwelle des 16. und 17. Jahrhunderts häufig Thema juristischer Dissertationen an mehreren Universitäten. ${ }^{27}$ Die Vorlage für die Abschriften der Gedichte aus dem Alten und Neuen Testament bei Tobias Hauschkonius konnten wir in der Nationalbibliothek nicht identifizieren, der Text selbst ist jedoch nicht unbekannt. ${ }^{28}$

Der Vergleich der überlieferten Vorlagen mit den poetischen Anverwandlungen war weniger aufschlussreich als erwartet. Eine Ausnahme bildet die versifizierte Magisterthese des Rochotius. Sein Gedicht zählt zudem zu den wenigen didaktischen Dichtungen aus dem Bereich der Rechtswissenschaft. Der Autor beginnt mit einer langen Einführung, in der er die Leser anspricht und den Kontext des Privilegs erklärt. Dieser Teil bildet etwa ein Drittel des gesamten Werkes. Dann paraphrasiert Rochotius den Text des Privilegs frei, nicht in der richtigen Reihenfolge, aber doch erkennbar. Das immer wiederkehrende Hauptmotiv ist die Dürftigkeit der Studenten, die des Wissens wegen in ein fremdes Land reisen. Genau so ein Student war Rochotius, der aus seiner Heimat in Oberungarn nach Prag reiste und dort auf die Hilfe von Mäzenen angewiesen war. Den Abschluss bildet eine vom Autor erfundene Belehrung über die Auswirkung dieses Privilegs - es soll sich auf Studenten, Magister und Professoren sowie auf gekrönte Dichter ${ }^{29}$ beziehen, hingegen sind Studenten, die faulenzen, in den Kneipen und mit Weibern ihr Leben vergeuden und mit ironischen Schriften andere beleidigen, von der Geltung des Privilegs ausgenommen. Wenn man die These von Rochotius mit anderen zeitgenössischen Thesen zum selben Thema vergleicht - alle waren in Prosa geschrieben -, so kann man die Unterschiede zwischen beiden Formen deutlich sehen: Bei Rochotius war die Form das Entscheidende, die in Prosa schreibenden Juristen mussten dagegen mehrere Argumente erfinden. Wie es bei den poetischen Prosavorlagen oft der Fall ist, waren die Fakten und Zitate, die nicht ins Metrum passten, am Rande ausgedruckt. Die

25 Diese Handschrift trägt die Signatur von Sebastian Tengnagel, sie musste also zu Campanus' Zeit schon in Wien gewesen sein, weil die Handschriften bis Nr. 7379 in den Jahren 1609-1610 zusammen geschrieben worden sind, vgl. Josef Stummvoll (Hg.), Geschichte der Österreichischen Nationalbibliothek, I, Wien 1968, S. 132.

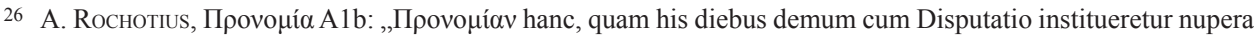
de Privilegiis studiosorum, chartis situ et tineis obsitis involutam reperi et errantem redegi, visum nunc in lucem dare idque sub tutela nominis vestri.“

27 Unmittelbar vor der These des Rochotius wurden drei Thesen in Frankfurt, Jena und Giessen veröffentlicht (VD17 1:013687M, 14:025472T, 12:158879T). Bei der These von Christoph CuPPENER von Logau, Commentarii ... in Authent. habita, C. ne Filius pro Patre, Frankfurt: Rotth 1605, wird schon auf der Titelseite wie bei Rochotius auf die Altertümlichkeit der Quelle hingewiesen: „ex vetustissima bibliotheca opus depromptum; nunc vero multis modis restitutum...".

28 Das Werk von Guido de Pileo (de Ferrara, Vicentinus) OP mit Inzipit Qui mundanam machinam potenter creas$t i$ befindet sich in Repertorium biblicum unter der Nr. 2756 (<http://www.repbib.uni-trier.de/cgi-bin/rebiIndex. tcl>; letzter Zugriff am 18. 7. 2019).

29 Rochotius selbst wurde 1606 zum poeta laureatus ernannt. Mehr RHB 4, S. 334-339. 
Sprache war voll von antiken Anspielungen und von Mythologie, so wird beispielsweise Kaiser Friedrich Barbarossa als „Ahenobarbus Athlas Friderici nomine gaudens“ bezeichnet. Ein Dichtung und ihre Vorlage vergleichendes Beispiel:

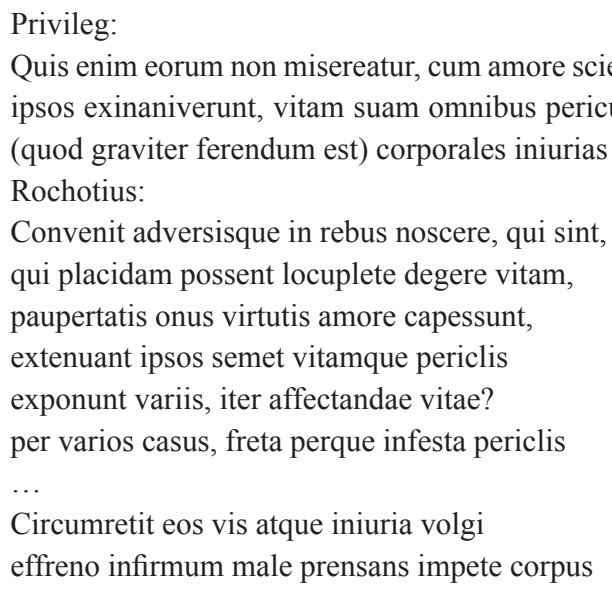

Anders verhält es sich bei Campanus. Dort, wo uns die Vorlagen für Campanus' Gedichten zur Verfügung stehen, handelt es sich um eher oberflächliche Nachahmung. Bei De epiphania wird die Predigt von Páleč in der Vorrede zitiert und in der Diskussion über die Richtigkeit der Bezeichnungen ,reges“ oder „magi“ benutzt. Der Text selbst zeigt kaum Einflüsse des Textes von Páleč. Bei dem Gedicht Querela pacis wird ein Zitat aus der Predigt von Simon von Tišnov auf der Titelseite abgedruckt, das Gedicht selbst zeigt jedoch keinerlei Ähnlichkeiten mit der angeblichen Vorlage; der symbolische Streit zwischen Krieg und Frieden ist eher der Tradition von Helius Eobanus Hessus und Ovids Episteln zuzuschreiben. Es ist bemerkenswert, dass die Passage in Simons Predigt Pace Troia nobilissima als einzige Stelle in der Predigt etwas mit der Antike zu tun hat, da dort über antike Städte gesprochen wird..$^{30}$ (Abb. 2) Entsprechend der Situation nimmt Campanus Prag aus der Aufzählung der Städte heraus - dort herrschte zu der Zeit kein Frieden. In Klammern erklärt er einzelne Wörter des zitierten Textes, die für den Leser nicht verständlich gewesen sein dürften. Es ist bedauerlich, dass die Vorlage für das Gedicht De nativitate verschollen ist. Die Struktur des Gedichts zeigt, dass es sich in diesem Fall tatsächlich um eine versifizierte Predigt handelt, deren Thema als Refrain immer wieder vorkommt. Campanus war sich des Unterschiedes zwischen dem mittelalterlichen und dem humanistischen Latein bewusst und änderte dementsprechend die Orthographie.

30 Pace insuper Troya nobilissima, Damascus opulentissima, Roma excellentissima, Karthago victoriosissima, Praga amenissima, Parisius (Lutetia) gloriosissima, creditur fuisse extructa. Templa deorum, civitates, urbes a maximis usque ad minimum tugur (tugurium) et quodlibet commune bonum historiacis leccionibus pacis legimus bonitate procurari. O pax desiderabile bonum, delectabile, amenum, jocundum, plenum deliciis, et altera Paradisus voluptatum! (transliteriert aus der Handschrift, in Klammern die Ergänzungen von Campanus, kursiv die Passagen, die Campanus ausließ). 


\section{Fazit}

Wie ist der Rückgriff der neulateinischen Dichter aufs Mittelalter zu erklären? Zunächst gab es hier die alte Tradition der Prager Universität, an die Campanus und seine Schüler bewusst anknüpften; die Tradition der Universität zu Zeiten von Jan Hus und seiner Zeitgenossen. Handschriften über die Geschichte Böhmens und Werke von Jan Hus fand man in der Frühen Neuzeit in vielen Gelehrtenbibliotheken. Weniger verbreitet war das Interesse für die Zeitgenossen von Hus, welches sich an der Auswahl der Texte von Campanus wohl dokumentieren lässt. Alle Autoren der anverwandelten Predigten sind Universitätsprofessoren aus vorhussitischer und hussitischer Zeit, einige zählten zunächst zu Hussens Anhängern und später zu seinen Gegnern. Auch wenn Campanus dies gewusst hätte, wäre das für ihn wahrscheinlich nicht entscheidend gewesen. Er war eher auf der Suche nach inspirativen Zitaten und Stoffen zu einem Thema, als dass er einen bestimmten Autor gesucht hätte, was ihn jedoch nicht daran hinderte, sich auf Autoren als Autoritäten zu berufen.

Mit seinem Interesse stand Campanus nicht alleine da - in vielen Handschriften mittelalterlicher Predigten aus dem Besitz der ehemaligen Prager Akademie in der Nationalbibliothek Prag findet man Marginalien inhaltlichen Charakters. Es handelt sich hierbei um zwei bis drei Hände, ${ }^{31}$ leider stammt keine Randbemerkung von Campanus' Hand, offensichtlich hatte er in die Handschriften nichts hineingeschrieben. Einer der Kommentatoren könnte Pavel Ješín gewesen sein, was aber noch stichhaltiger bewiesen werden müsste. ${ }^{32}$ Von ihm, einem Schüler von Campanus und Herausgeber der Maiestas Carolina und der Dalimil-Chronik, weiß man, dass er sich Handschriften aus der Universitätsbibliothek auslieh, wobei es fraglich bleibt, ob er sie auch alle zurückgegeben hat.

Die Tradition der Prager Universität wurde außerdem durch verschiedene Privilegien symbolisiert, was sich auch in den Werken von Exulanten wie Martinius und Hauschkonius widerspiegelt. Welche Bedeutung alte Dokumente für die Universitätsmitglieder hatten, kann man den Propstbüchern entnehmen: Als 1608 das Türmchen auf dem Karlskolleg nach einem Gewitter wackelte, hat man es heruntergetragen und repariert. Im Innern des Türmchens befanden sich verschiedene Dokumente, darunter auch das Schreiben der Konstantinopolitanischen Kirche an die Prager Universität (aus der gedruckten Ausgabe von Caspar Nydbruck kopiert) und deren Antwort. Bevor man diese in das bereits reparierte Türmchen zurücklegte, kopierte man ihren Text in das Propstbuch und fügte noch einige neue Dokumente bei, darunter die Academiae Pragensis fortuna genannte Kurzgeschichte der Universität. ${ }^{33}$ Auch das ist ein Zeugnis für die Einstellung der Universitätsmitglieder zu den Quellen der Universitätsgeschichte.

Mit dem bereits erwähnten Kaspar Nydbruck und seinen Kontakten zu böhmischen Humanisten hängt das langjährige Interesse für alte Dokumente und Kodizes zusammen.

31 Ähnliche Marginalien findet man Michal Dragoun zufolge z. B. in den Handschriften X B 2, IV C 18, X C 25 (Handschriften aus dem Kolleg der Böhmischen Nation), IV G 26, X B 17, III A 24 (Handschriften aus dem Karlskolleg), IV A 12, IV D 2 (Lauda-Kolleg).

32 Wir haben mit bekannten Autographen verglichen, die sich in der Handschrift der Bibliothek des Nationalmuseums Prag XVII D 25 und in dem Konvolut historischer Drucke II 17.683 in der Wissenschaftlichen Bibliothek in Olmütz befinden (für die Kopien danken wir Rostislav Krušinský). Über die Unsicherheit bei der Identifizierung des Autographs von Ješín s. Jiří DAŇHELKA, Ješínovy výpisky z Dalimilovy kroniky [Ješíns Exzerpte aus der Dalimil-Chronik], Listy filologické 84, 1961, S. 58-59.

33 Acta praepositorum, Fol. 587v-592r, auf 591r-592r Academiae Pragensis fortuna. 
Es war Nydbruck, der die Handschriften von Motten befreite und zum Vorschein brachte; sein „situ et tineis“ findet man auch in der Vorrede von Rochotius, und in ähnlichem Sinne redet Campanus in seinem Abschlussgedicht zur Ausgabe von Nemo seinen Schüler Martin Mylius an. ${ }^{34}$ Was im 16. Jahrhundert ernst gemeint war, wurde zu Beginn des 17. Jahrhunderts zu einem modischen Topos, indem man verschiedene Quellen, die in gedruckten Exemplaren sehr wohl zugänglich waren, als alte Dokumente schilderte, die endlich zum Vorschein gebracht wurden.

Es stellt sich noch die Frage, zu welcher Zeit und welcher Gelegenheit die Paraphrasen und Ausgaben der mittelalterlichen Werke publiziert wurden. Im Fall der These von Rochotius ist anzunehmen, dass seine Themenwahl mit der Universitätsreform zusammenhing. Seiner Vorlage entnahm er die Idee, dass die Studenten im Rahmen der Universität systematisch gefördert werden müssen. Gleichzeitig verfolgte er seine eigenen Ziele: sich bei seinen Mäzenen für die konkrete Unterstützung zu bedanken, wozu ihm das Privileg den passenden Stoff lieferte.

Bei Campanus sollte hervorgehoben werden, dass alle obengenannten Werke 1618 erschienen. Einige wurden zu Jahresbeginn verfasst und als Neujahrsgeschenk an Freunde und Mäzene verteilt. Sie spiegeln sein Interesse an den mittelalterlichen Texten seiner Vorgänger an der Universität wider, deren Stil er als „unschuldige Schlichtheit“ bezeichnete. Offensichtlich fand er die mittelalterliche Ausdruckweise, die sich nicht der antiken Mythologie bediente, als veraltet. Nicht die Form, sondern der Inhalt der mittelalterlichen Predigten war für ihn wichtig. Anders verhielt es sich bei den mittelalterlichen Versformen, die Campanus zweifellos stark beeinflusst haben. In seiner metro-rhythmischen Dichtung vereinigten sich mittelalterliche Dichtung und Leoninische Hexameter, studentische gereimte Dichtung, die stets als Unterströmung an den Universitäten existierte, und die tschechische gereimte religiöse Dichtung, deren Tradition im Lateinischen bis hin zu Wenceslaus Nicolaides Vodňanský zurückverfolgt werden kann.

Eine Aktualisierung älterer Predigt stellt das Gedicht Querela pacis dar. Es entstand zu Beginn des Böhmischen Krieges nach dem zweiten Prager Fenstersturz. Campanus nutzte die Predigt von Simon von Tišnov, die - so Campanus - 200 Jahre zuvor an König Wenceslaus gerichtet war, bewusst in der Zeit nach dem ersten Fenstersturz, der den Beginn der Hussitenkriege bedeutete. ${ }^{35} \mathrm{Er}$ fürchtete eine Wiederholung der Geschichte und mahnte alle Konfliktbeteiligten zur Versöhnung. Als echter Humanist war er ein entschlossener Kritiker von Kriegen.

Als Bekenntnis zur Tradition, vielleicht auch als nostalgische Erinnerung der Exulanten an die alma mater, die es nicht mehr gab, und nicht zuletzt als möglichen Beweis, dass nach 1620 einige Handschriften die Universitätsbibliothek verließen, kann man die Ausgabe der mittelalterlichen Gedichte aus dem Kodex der Karlsuniversität bei Tobias Hauschkonius und den Abdruck des Kuttenberger Dekrets von 1409 und des Mandats zur Übergabe der

34 J. Campanus, Nemo, S. A4a: „Quem legis, in tenebris et carcere Nemo latebat / nunc patet et rutilum spectat in axe jubar / Vix aeger tamen incedit sermone pedestri, / hoc vitium longa traxerat ille mora. / Ut Stylus addat opem misero, qua nutat, equester / Est opus huic opera, quam rogat ipse, tua / Si sic perfectus numeris erit omnibus ille / debebit numeris omnia puncta tuis.“

35 Otakar Odložilík datierte die Predigt in das Jahr 1420 und vermutete als Adressaten Kaiser Sigismund, s. O. Odložı́í, Z počátků husitství na Moravě [Von den Anfängen des Hussitentums in Mähren], Brno 1927, S. $127-128$. 
Karlsuniversität an das im Prager Klementinum siedelnde Jesuitenkolleg in Parentatio von Samuel Martinius werten. Die Privilegien und wichtige Dokumente hatten mit ihrem Bezug zur Universität stets symbolische Bedeutung und trugen, abgedruckt in Büchern, zu Autorität und Authentizität bei. ${ }^{36}$

Das Interesse für alte Handschriften und Dokumente war in den Böhmischen Ländern nicht allein eine Angelegenheit protestantischer Gelehrter. Aus tschechischem Milieu können gleich zwei Namen katholischer Intellektueller genannt werden, die sich für die Geschichte Böhmens und für die mittelalterliche Tradition interessierten. An erster Stelle ist Johann Bartholdus Pontanus von Breitenberg zu nennen, ein passionierter Büchersammler (und u. a. Käufer alter Bücher aus Universitätsbesitz) und Herausgeber der Statuta provincialia Arnesti. Weiter sollte Jan Sixti von Lerchenfels erwähnt werden, der z. B. ein altes gereimtes Benedicamen ins Tschechische übersetzte und drucken ließ. Für die Katholiken bedeutete das Mittelalter allerdings die Rückkehr in jene Zeit, da die Böhmischen Länder noch ausschließlich zur katholischen Kirche gehörten.

(Sprachliche Korrektur: Wolf B. Oerter)

Abbildungen s. auf den Seiten 281-284

\section{Danksagung und finanzielle Förderung}

Der Aufsatz entstand im Rahmen des Projekts GA16-09064S „Podoby humanismu v literatuře českých zemí - Formen des Humanismus in der Literatur der Böhmischen Länder (1469-1622)“ der Tschechischen Agentur zur Förderung der wissenschaftlichen Forschung (Grantová agentura České republiky). - Für die Mitarbeit an diesem Beitrag bin ich Michal Dragoun zu Dank verpflichtet.

MARTA VACULÍNOVÁ

\section{DIGNA FAVORE SIMPLICITAS. STŘEDOVĚKÉ RUKOPISY JAKO PŘEDLOHA PRO LATINSKÉ BÁSNĚ JANA CAMPANA A JEHO ŽÁKŮ}

\section{RESUMÉ}

Pro rozvoj výuky historie na pražské univerzitě počátku 17. století byla rozhodující osobnost Jana Campana. Jako historik-analista nebyl Campanus v moderním bádání př́liš kladně hodnocen, větší význam bývá přisuzován jeho pedagogickému působení. Díky němu vyšli z pražského učení historici a editoři jako Pavel Ješín nebo Pavel Stránský ze Zhoře. Campanus zadával svým žákům ke zpracování témata z národních i regionálních dějin, nejčastěji básnickou formou, kterou preferoval i on sám. Prameny hledal i v univerzitním archivu a rukopisech kolejních knihoven a vedl $\mathrm{k}$ tomu i své žáky. V období mezi univerzitní reformou a Bílou horou tak vzniklo několik básnických skladeb, inspirovaných středověkými rukopisy z univerzitních sbírek. Sám si vybíral především

36 Über die Gedenkschriften im Rathausturm der Prager Neustadt im Jahre 1612 ähnlich Jaroslava MendelovÁ, Jan Campanus Vodñanský ve fondech pražského městského archivu [Johannes Campanus Wodnianus in den Beständen des Prager Stadtarchivs], Acta Universitatis Carolinae - Philosophica et Historica 5, Z pomocných věd historických XIV, 1996, S. 155-161. 
kázání univerzitních profesorů počátku 15. století (Šimon z Tišnova, Petr Koněprus, Štěpán z Pálče), zatímco Campanův žák Ondřej Rochotius zpracoval veršem privilegium Friedricha Barbarossy pro univerzitní studenty. Část rukopisných předloh se podařilo identifikovat v Národní knihovně v Praze. Díky nim bylo možné provést srovnání předloh a jimi inspirovaných básnických textů. Středověká kázání zaujala Campana zejména svým obsahem, formu považoval spíše za zastaralou. Vyhledával náměty, které byly přses velký časový odstup stále aktuální (ohrožení míru, kritika mravů společnosti). Ne náhodou většina takto inspirovaných skladeb vznikla v roce 1618, kdy začala česká válka.

Mgr. Marta Vaculínová, Ph.D.

Kabinet pro klasická studia Filosofického ústavu AV ČR

(Kabinett für Klassische Studien des Philosophischen Instituts der Tschechischen AdW)

vaculinova@ics.cas.cz 